\title{
Accuracy of contrast-enhanced ultrasound compared with conventional ultrasound in acute pancreatitis: Diagnosis and complication monitoring
}

\author{
DIMING CAI ${ }^{1}$, SHYAM SUNDAR PARAJULY ${ }^{1}$, HUIYAO WANG $^{2}$, XIAOLING WANG $^{3}$, \\ WENWU LING ${ }^{1}$, BIN SONG ${ }^{4}$, YONGZHONG LI ${ }^{1}$ and YAN LUO ${ }^{1}$ \\ ${ }^{1}$ Department of Ultrasound, West China Hospital; ${ }^{2}$ Training Department of Resident Doctor, \\ West China School of Medicine; Departments of ${ }^{3}$ Operations Management and ${ }^{4}$ Radiology, \\ West China Hospital, Sichuan University, Chengdu, Sichuan 610041, P.R. China
}

Received June 24, 2015; Accepted September 1, 2016

DOI: $10.3892 / \mathrm{etm} .2016 .3760$

\begin{abstract}
Contrast-enhanced ultrasound (CEUS) has been used for diagnosing acute pancreatitis (AP), particularly severe acute pancreatitis (SAP). However, the diagnostic difference between CEUS and conventional ultrasonography (CUS) for AP and SAP has not been reported. The aim of the present study was to investigate the diagnostic accuracy of CUS and CEUS for AP. A total of 196 patients clinically diagnosed with AP were selected. All patients underwent CUS, CEUS and contrast-enhanced computed tomography (CECT) within 72 h. CECT was considered the gold standard. Pancreatic size, peripancreatic fluid collection (PPFC) and splenic vessel complications were the variables observed by CUS and CEUS. The differences in the variables among the three methods were analyzed using the $\chi^{2}$ test and statistical analysis software. Significant differences in pancreatic size, PPFC and splenic vessel complications in AP were observed between CEUS and CUS $(\mathrm{P}<0.05) \cdot \chi^{2}$ test results indicated that CEUS significantly differed from CUS in terms of having a higher diagnostic accuracy for AP and SAP $(\mathrm{P}<0.05)$. The results indicate that CEUS is a reliable method for the diagnosis and monitoring of AP and SAP, and may be substituted for CECT.
\end{abstract}

\section{Introduction}

Acute pancreatitis (AP), which can be divided into mild acute pancreatitis (MAP) and severe acute pancreatitis (SAP), is an inflammatory disorder of the pancreas that has been clinically

Correspondence to: Dr Yan Luo, Department of Ultrasound, West China Hospital, Sichuan University, 37 Guoxue Lane, Chengdu, Sichuan 610041, P.R. China

E-mail:yanluodoc@163.com

Key words: acute pancreatitis, severe acute pancreatitis, conventional ultrasound, contrast-enhanced ultrasound, peripancreatic fluid collection, splenic vessel complications, diagnosis defined as a common form of acute abdominal pain (1-3). MAP has a good prognosis and few complications with low mortality. However, SAP accompanied by serious complications is reported to have a high mortality rate (4). Although SAP accounts for only $15-25 \%$ of AP cases, its mortality rate may be as high as $15-48.4 \%$ (5). Early diagnosis and evaluation of the severity of pancreatitis is crucial in AP management $(6,7)$.

Ultrasonographic examination is a medical diagnostic imaging modality that is commonly used for AP because of its convenience, portability, safety in terms of radiation exposure, and low cost. Biliary tract disease, such as gallstones, is the main etiological factor leading to AP in China (8). Conventional ultrasonography (CUS) is considered to be a useful diagnostic imaging modality for AP because it not only is able to detect pancreatic and peripancreatic conditions, but also can be used to investigate whether biliary tract disease has led to the occurrence of AP (9).

However, the diagnostic sensitivity of CUS for SAP is reported to be low (6). It is also unclear whether CUS is more important for diagnosing AP or SAP. Some studies have shown that contrast-enhanced ultrasonography (CEUS) has become an important diagnostic method for SAP because it is able to detect necrosis of the pancreatic parenchyma without the hepatic and renal toxicity possible with contrast-enhanced computed tomography (CECT) $(10,11)$. If a difference in accuracy between CUS and CEUS for the diagnosis of AP and SAP exists, CEUS examination should be routinely applied instead of CUS to diagnose AP or SAP.

Pancreatic size, peripancreatic fluid collection (PPFC), and splenic vessel complications are the variables observed on CUS and CEUS that are accepted to be important for assessing AP. CECT is the gold standard imaging modality for the diagnosis of AP (12), particularly for SAP, but some research studies have found that SAP can be successfully diagnosed using CEUS (13). A previous study reported that a diagnosis of SAP made using CEUS was strongly correlated with that made using CECT (14). It has been demonstrated that CEUS has a higher diagnostic value than CUS for $\operatorname{AP}$ or $\operatorname{SAP}(15,16)$.

However, the specific differences between CUS and CEUS in terms of the diagnostic sensitivity for AP or SAP 
have not been clarified in the literature. Therefore, the present study aimed to investigate whether there any statistically significant differences between CUS and CEUS, and between CECT and CEUS in terms of diagnostic accuracy for AP or SAP. In addition, it aimed to investigate the difference in diagnostic accuracy for AP and SAP between CUS and CEUS in comparison with that of CECT as the gold standard. This study focused on differences between imaging with different types of US. The clinical and laboratory factors associated with AP were not considered in this study.

\section{Materials and methods}

Patient data. This study was a prospective study. It was conducted in accordance with the Declaration of Helsinki, and with approval from the Ethics Committee of Sichuan University (Chengdu, China). Written informed consent was obtained from all participants. In total, 220 inpatients given a clinical diagnosis of AP at Sichuan University from May 2011 to December 2012 were selected for this study. The inclusion criteria were: Patients undergoing CUS, CEUS and CECT examinations without any contraindications. The pancreas was displayed clearly on CUS. Exclusion criteria: Any AP patients who could not undergo the three examinations, or patients for which the pancreas could not be distinctly shown by US. According to the criteria, 5 patients had to be excluded from the study because sonographic imaging of the pancreas was impaired by overlying bowel gas prohibiting visualization of the pancreas during the US and CEUS examinations; 3 patients were excluded because of pain that made examination impossible; 10 patients were excluded because they refused to undergo CEUS examination; 4 patients had a contraindication to administration of the ultrasound contrast medium SonoVue due to coronary heart disease; and 2 patients were excluded because of contraindications to computed tomography (CT) with contrast injection (renal failure or known allergy to iodinated contrast medium). Finally, 196 patients constituted the study population.

All patients were examined using CUS and pancreatic CEUS in order to confirm the AP diagnosis and, if confirmed, whether it was MAP or SAP. All ultrasonographic examinations were performed within $72 \mathrm{~h}$ of admission. All CECT examinations were performed within $4 \mathrm{~h}$ of the ultrasound (in all cases within $72 \mathrm{~h}$ after the onset of symptoms). The sonographer and radiologist were blinded to other laboratory or imaging findings, respectively.

Sonographic examination. The LOGIQ E9 ultrasonographic system (GE Healthcare, Milwaukee, WI, USA) with a C1-5-MHz corresponding probe or the PHILIPS iU22 (Philips Medical Systems, Bothell, WA, USA) ultrasonographic system with a corresponding C5-2-MHz transducer was used. The two ultrasonographic systems were each equipped with harmonic contrast pulse sequencing technology. The contrast agent used was SonoVue (Bracco SpA, Milan, Italy), which is a suspension of stabilized sulfur hexafluoride microbubbles.

Two sonographers who had $>10$ years' experience with CUS of the abdomen and $>3$ years' experience with CEUS evaluation for pancreatic disease were chosen to perform the examinations. All patients fasted for $8 \mathrm{~h}$ prior to undergoing the ultrasonographic examination. First, grayscale sonography and color Doppler ultrasonography were performed in order to observe the pancreas volume or changes in parenchymal structure. The PPFC, including the bursa omentalis and peripancreas interspaces, particularly the bilateral pararenal space; the anterior pararenal space; and the posterior pararenal space were all examined. Any splenic vessel complications, including splenic artery embolism, splenic artery stenosis, splenic artery aneurysms, splenic vein embolism, and splenic vein stenosis were observed. The CUS results were recorded.

A different sonographer then started the CEUS program at a low mechanical index (LOGIQ E9, 0.12; PHILIPS iU22, 0.06). A 1.5-2.0 ml volume of contrast agent suspension (SonoVue) was administered as a bolus injection through the antecubital vein, followed by flushing with $5 \mathrm{ml}$ saline solution. After an 8-15 sec delay, the pancreatic parenchyma began to undergo enhancement. The real-time contrast-enhanced image obtained after contrast agent injection was recorded on a hard disk, and the injection times were calculated simultaneously. In the entirely arterial pancreatic and splenic systems, the contrast phases were identified as the artery phase (0-30 sec after contrast agent injection) and venous phase (starting at $31 \mathrm{sec}$ after contrast agent injection). The results of the pancreatic CEUS with pancreas, PPFC, and splenic vessel complications were recorded.

CT examination. For this study, 64-slice spiral CT (Philips Brilliance; Philips Medical Systems, Cleveland, OH, USA) or 18-slice spiral CT (Somatom Sensation 16; Siemens, Erlangen, Germany) was used. The contrast agent was iopamidol (Iopamiro; Bracco SpA) or iopromide (Ultravist; Bayer Schering Pharma AG, Leverkusen, Germany) at a concentration of $37 \mathrm{gI} / 100 \mathrm{ml}$.

The range of CECT scanning was from the chest to the pelvic floor. A total amount of 90-120 $\mathrm{ml}$ iopamidol or iopromide was injected into each patient via the antecubital vein at a rate of $3 \mathrm{ml} / \mathrm{sec}$ using a power injector.

Standard of diagnosis. Physicians with $>5$ years of experience with abdominal imaging readings interpreted the results. The physicians were blinded to the ultrasonographic imaging results. First, an increase in pancreas volume and changes in parenchymal structure were assessed using CECT, which was considered as the standard diagnostic imaging modality for AP. The PPFC and splenic vessel complications were then assessed. Finally, the pancreatic parenchyma was assessed for pancreatic necrotic lesions, the Balthazar grade was determined (17), and a diagnosis of SAP was made accordingly. The standard indicators for SAP diagnosis included the presence of a non-enhancing area in the pancreas and/or a Balthazar severity grade D or E. The minimum size of heterogeneity or decreased enhancement area with a pancreatic necrotic lesion was $1 \mathrm{~cm}$.

The sonographic films were read by two sonographers. First, the CUS films were read by two experienced sonographers with $>10$ years' experience with abdominal ultrasonography who were blinded to other laboratory or imaging findings. They evaluated the images for an increase in pancreas volume, changes in the parenchymal structure with a hypoechoic appearance (compared with the echo of the surrounding liver) and whether the echogenicity was uniform. 
Table I. Results of observations by CECT, CUS and CEUS and the rate of diagnosis in the total study population.

\begin{tabular}{|c|c|c|c|c|}
\hline Observations & $\begin{array}{l}\text { Examination } \\
\text { results }\end{array}$ & CECT, n (\%) & CUS, n (\%) & $\begin{array}{c}\text { CEUS, } \\
\mathrm{n}(\%)\end{array}$ \\
\hline \multirow[t]{2}{*}{ Parenchyma with enlarged pancreas } & Homogeneous & $77(39.3)$ & $83(42.3)$ & $71(36.2)$ \\
\hline & Inhomogeneous & $45(23.0)$ & $65(33.2)$ & $39(19.9)$ \\
\hline \multirow[t]{2}{*}{ Parenchyma with no enlarged pancreas } & Homogeneous & $12(6.1)$ & $29(14.8)$ & $20(10.2)$ \\
\hline & Inhomogeneous & $62(31.6)$ & $19(9.7)$ & $66(33.7)$ \\
\hline \multirow[t]{2}{*}{ Peripancreatic fluid collection } & Positive & $178(90.8)$ & $151(77.0)$ & $172(87.8)$ \\
\hline & Negative & $18(9.2)$ & $45(23.0)$ & $24(12.2)$ \\
\hline \multirow[t]{2}{*}{ Splenic vessel complications } & Positive & $51(26.0)$ & $12(6.1)$ & $30(15.3)$ \\
\hline & Negative & $145(74.0)$ & $181(92.3)$ & $170(86.7)$ \\
\hline
\end{tabular}

Percentages were calculated for the total study population $(n=196)$. CECT, contrast-enhanced computed tomography; CUS, conventional ultrasonography; CEUS, contrast-enhanced ultrasonography.

The criteria for a normal pancreas on ultrasonography were that the head of pancreas was not $>2.5 \mathrm{~cm}$ and the body or tail of the pancreas was not $>2.0 \mathrm{~cm}$. On a transverse section, the pancreas anteroposterior diameter was used as the size of the pancreatic head, which was measured adjacent to the portal vein. The body of the pancreas was measured at the position of the anterior of the aorta abdominalis on a transverse section. The tail of the pancreas was measured at the hilus lienis on an oblique transverse section.

The sonographers made a diagnosis of SAP according to peripancreatic or retroperitoneal space effusion, the change in pancreatic parenchymal echogenicity, the continuous status of the pancreatic capsule, the pancreatic parenchyma necrosis, and splenic vessel complications observed with color Doppler technology. All results were recorded in the computer.

The sonographers then read the CEUS films to search for signs of pancreatic swelling, pancreatic parenchyma necrosis, PPFC including the bursa omentalis, retroperitoneal space effusion, and/or local complications such as necrotic tissue, abscess and pancreatic pseudocysts. The CEUS diagnostic standard indicators of SAP were the same criteria with a Balthazar CT score to measure the severity of AP, and included the presence of pancreatic necrosis and/or one or more PPFCs and/or complications. Splenic vessel complications were observed and recorded using CEUS.

Statistical analysis. The disease severity was established according to the aforementioned CECT criteria. $\mathrm{P}<0.05$ was considered to indicate a statistically significant result. SPSS version 18.0 software (SPSS, Inc., Chicago, IL, USA) with the Pearson $\chi^{2}$ test used to analyze the observations, including the pancreatic size, PPFC and splenic vessel complications, with pairwise comparisons between CECT, US and CEUS. The $\chi^{2}$ test was used to analyze the diagnostic accuracy of CUS and CEUS for AP and SAP.

\section{Results}

Results obtained using CECT, US, and CEUS. There were 196 patients [129 men and 67 women; age (mean \pm standard deviation), 48.1 \pm 13.9 years; range, 18-79 years]. included in the study. All cases were inpatients at West China Hospital, Sichuan University.

According to the design of experiments, the gold standard is CECT at $72 \mathrm{~h}$ for SAP with Balthazar's severity grade. CUS criteria for AP were: The echogenicity was hypoechoic, the pancreatic volume was enlarged, which was indicative of swelling (the head of the pancreas was $>2.5 \mathrm{~cm}$ or the body or tail was $>2.0 \mathrm{~cm}$ ) and PPFC was found surrounding the pancreas or the retroperitoneal space effusion. The CUS criteria for SAP were: In addition to the AP criteria, pancreatic parenchymal necrosis or splenic vessel complications detected using color Doppler. The CEUS criteria for AP were: Pancreatic volume was enlarged (the head of the pancreas was $>2.5 \mathrm{~cm}$, the body or tail of the pancreas was $>2.0 \mathrm{~cm}$ ), or the continuous status of the pancreatic capsule was interrupted. PPFC was found in the peripancreatic or retroperitoneal space effusion. The CEUS criteria for SAP were: Necrosis of the pancreatic parenchyma was detected and splenic vessel complications were observed. The degree of necrosis was evaluated, and categorized as $<30 \%, 30-50 \%$ or $>50 \%$.

By CECT, 122 and 74 patients were diagnosed with and without pancreatic swelling, respectively. In addition, PPFC was detected in 178 patients, and splenic vessel complications were found in 51 patients. By CUS, 148 patients were diagnosed with pancreatic swelling, 151 with PPFC, and 12 with splenic vessel complications. By CEUS, 110 patients were diagnosed with pancreas swelling, 172 with PPFC, and 30 with splenic vessel complications (Table I).

Statistical data for pancreatic size obtained using the three modalities. For pancreatic volume in the same section determined using the three methods, statistically significant differences were observed among the three methods $\left[\chi^{2}=43.227\right.$, degrees of freedom $\left.(\mathrm{df})=2, \mathrm{P}=0.0000001\right)$. CECT and CEUS significantly differed from CUS (CECT vs. CUS: $\chi^{2}=33.737, \mathrm{df}=1, \mathrm{P}=0.0000002$; CEUS vs. CUS: $\chi^{2}=35.076$, $\mathrm{df}=1, \mathrm{P}=0.0000001)$. However, no significant difference was observed between CECT and CEUS $\left(\chi^{2}=2.797, \mathrm{df}=1, \mathrm{P}=0.424\right.$; 

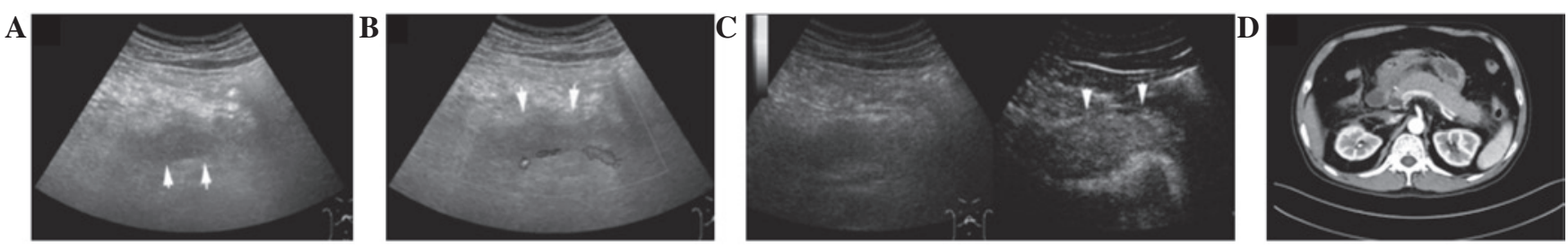

Figure 1. Imaging results for a 42-year-old man with acute pancreatitis. (A and B) Gray scale ultrasonography and color Doppler ultrasonography (conventional ultrasound) revealed that the pancreatic parenchyma was hypoechoic and the pancreatic boundary was blurred (solid arrows). (C) Contrast-enhanced ultrasonography clearly showed that the pancreatic parenchyma was swollen and the pancreatic boundary was sharp-edged (solid arrow). (D) Contrast-enhanced computed tomography showed that the pancreatic parenchyma was enhanced and the pancreatic boundary was sharp-edged.
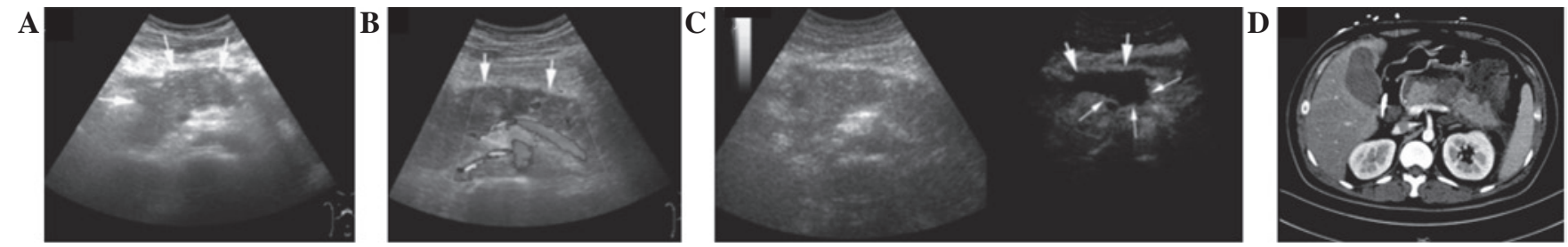

Figure 2. Imaging results for a 35-year-old man with severe acute pancreatitis. (A and B) Gray scale ultrasonography and color Doppler ultrasonography (conventional ultrasound) showed that the echo of the pancreatic parenchyma was hypoechoic and inhomogeneous, and there was no obvious peripancreatic fluid collection or necrosis of the pancreatic parenchyma (solid arrow). (C) A necrotic lesion with an extent of $\sim 2 \times 1 \mathrm{~cm}^{2}$ with no enhancement was displayed in the pancreatic parenchyma (small solid arrow) and an irregularly ranged area of peripancreatic fluid collection with no enhancement was displayed in the pancreatic omental bursa by contrast-enhanced ultrasound (big solid arrow). (D) contrast-enhanced computed tomography showed that necrosis of the pancreas was present with no enhancement.
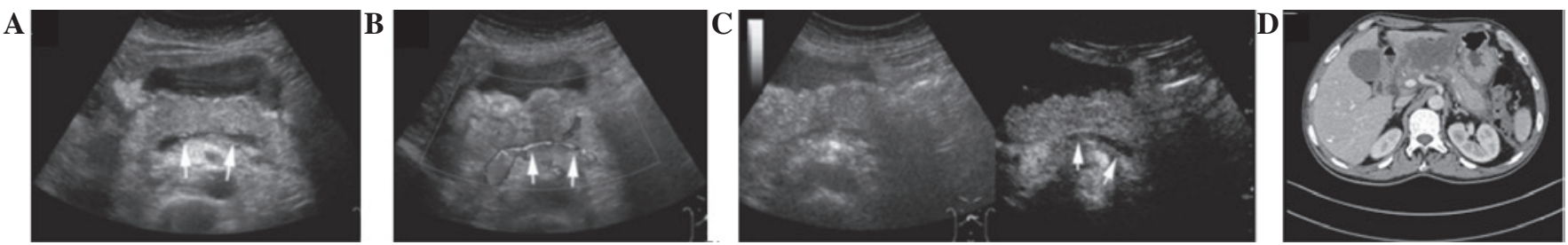

Figure 3. Imaging results for a 63-year-old woman with severe acute pancreatitis. (A and B) The splenic vein stenosis behind the pancreas was displayed by gray scale ultrasonography (solid arrow). Blood flow stenosis of the splenic vein was diaplayed by color Doppler ultrasonography (solid arrow). (C) The patient was diagnosed with splenic vein embolism because there was no enhancement of the splenic vein by contrast-enhanced ultrasonography (solid arrow). (D) Contrast-enhanced computed tomography showed that the splenic vein was stenotic with partial embolism.

Fig. 1). $\mathrm{P}<0.05$ was considered to indicate a statistically significant difference.

Statistical data for PPFC obtained using the three modalities. For PPFC, statistically significant differences were observed among the three methods $\left(\chi^{2}=16.269, \mathrm{df}=2, \mathrm{P}=0.0003\right)$. CECT and CEUS significantly differed from CUS (CECT vs. CUS: $\chi^{2}=13.787, \mathrm{df}=1, \mathrm{P}=0.0002$; CEUS vs. CUS: $\chi^{2}=7.757, \mathrm{df}=1$, $\mathrm{P}=0.005)$. However, no significant difference was observed between CECT and CEUS ( $\chi^{2}=0.960, \mathrm{df}=1, \mathrm{P}=0.327$; Fig. 2 ).

Statistical data for splenic vessel complications obtained using the three modalities. For splenic vessel complications, including splenic artery and vein complications such as splenic artery embolism, splenic vein embolism, splenic artery stenosis, splenic vein stenosis, and splenic artery aneurysms, statistically significant differences were observed among the three methods $\left(\chi^{2}=29.199, \mathrm{df}=2, \mathrm{P}=0.0000004\right)$. CECT significantly differed between CEUS and CUS (CECT vs.
CUS: $\chi^{2}=28.766, \mathrm{df}=1, \mathrm{P}=0.00000008, \mathrm{CECT}$ vs. CEUS: $\left.\chi^{2}=6.862, \mathrm{df}=1, \mathrm{P}=0.009\right)$. CEUS significantly differed from CUS $\left(\chi^{2}=8.640, \mathrm{df}=1, \mathrm{P}=0.003\right.$; Fig. 3 ).

Statistical data for AP and SAP diagnosis using the three modalities. Among the 196 patients, 132 were diagnosed with SAP by CECT and 64 patients were diagnosed with MAP. By CUS, 167 were diagnosed with AP and 63 were diagnosed with SAP. By CEUS, 184 patients were diagnosed with AP and 103 with SAP (Tables II and III). Analysis using $\chi^{2}$ tests, revealed a significant difference between CEUS and CUS in terms of AP $\left(\chi^{2}=7.872, \mathrm{df}=1, \mathrm{P}=0.005\right)$ and SAP diagnoses $\left(\chi^{2}=25.965, \mathrm{df}=1, \mathrm{P}=0.0000003\right)$.

\section{Discussion}

Medical imaging is an important diagnostic method for AP. Ultrasonography is widely used in AP diagnosis (18). In the present study, among the 196 patients, there were 167 
Table II. Diagnosis of AP by CUS and CEUS.

\begin{tabular}{lccc}
\hline Imaging & Positive & Negative & Total \\
\hline CUS & 167 & 29 & 196 \\
CEUS & 184 & 12 & 196 \\
Total & 351 & 41 & 392
\end{tabular}

Results shown are for the total study population $(n=196)$. AP, acute pancreatitits; CUS, conventional ultrasonography; CEUS, contrast-enhanced ultrasonography.

Table III. Diagnosis of SAP by CUS and CEUS.

\begin{tabular}{lccc}
\hline Imaging & Positive & Negative & Total \\
\hline CUS & 63 & 69 & 132 \\
CEUS & 103 & 29 & 132 \\
Total & 166 & 98 & 264 \\
\hline
\end{tabular}

Results shown are for the patients in the study population that were diagnosed with SAP by contrast-enhanced computed tomography $(n=132)$. SAP, severe acute pancreatitits; CUS, conventional ultrasonography; CEUS, contrast-enhanced ultrasonography.

and 184 patients diagnosed with AP using CUS and CEUS, respectively. The results for diagnosis by CEUS were closer than those of CUS to the diagnosis results obtained using the gold standard of CECT. This demonstrates that CEUS is a more convincing method than US for AP and SAP diagnosis. The results conformed with those reported in previous studies, that CEUS produces excellent results in the staging of acute pancreatitis severity $(10,12,14-16)$.

Pancreatic swelling is an important feature of AP (19). In comparison with the results obtained using CECT, the diagnostic rates with CUS and CEUS were 121\% (148/122) and 91\% (111/122), respectively. These results indicate the CUS overestimated the pancreatic size and thus did not reflect the real size of the pancreas. One reason for this may be that CUS barely differentiates the boundary of the pancreas. In particular, when PPFC appeared in the lesser peritoneal sac within hemorrhage or abscess with hypoecho there was no boundary between the PPFC and edema of the pancreatic parenchyma, which also appears hypoechoic by CUS.

PPFC often occurs after the onset of AP (20). In comparison with the results obtained using CECT, the CUS diagnostic rate of PPFC was $84.8 \%$ (151/178). With CEUS, the diagnostic rate was $96.6 \%(172 / 178)$, and the results were closer to those for CECT. Since the quality of pancreatic fluid collection varies, echogenic features also vary. CEUS is more specific than CUS in displaying the pancreatic parenchyma edema, the border-capsula of the pancreas, the collection fluid of the peripancreas, and the peripancreatic necrosis. A previous study indicated that CUS hardly differentiated between PPFC and pancreatic parenchymal swelling (21).

In the present study, the results confirmed that CUS overestimated pancreatic size and underestimated pancreatic fluid collection. By contrast, the pancreatic parenchyma was enhanced on CEUS. An ultrasonographic finding of pancreatic parenchymal necrosis exhibits no enhancement area following the injection of contrast agent. This result in PPFC concurs with that reported by Golea et al (22).

Peripancreatic vessel complications of AP have low morbidity $(23,24)$. They include splenic vessel, portal vein and mesenteric vessel complications $(25,26)$. Most patients with splenic vessel complications in AP present with few clinical symptoms, but such complications may lead to severe, sometimes fatal, outcomes, such as splenic artery aneurysm rupture (27). In this study, splenic vessel complications were examined; splenic artery and vein complications such as splenic artery embolism, splenic vein embolism, splenic artery stenosis, splenic vein stenosis, and splenic artery aneurysms are examples of the complications (28-30).

In the present study, all patients with splenic vessel complications were from the SAP group. Studies concerning the use of CUS in the diagnosis of splenic vessel complications are few (31). Since the peripancreatic vessels are located in the posterior peritoneum, echo attenuation is the main factor that affects diagnosis; however, the results of grayscale sonography are greatly affected by meteorism in the gastrointestinal tract, and diagnosis is sometimes difficult (32). In the present study, the diagnostic rates with CUS and CEUS were 23.5\% (12/51) and 58.8\% (30/51), respectively. The diagnostic yield of CUS is lower than that of CEUS (23.5\% vs. $58.8 \%)$. The reasons why ultrasonography often resulted in missed diagnoses of splenic vessel complications require consideration. The ultrasonographic result would have been greatly affected by meteorism in the gastrointestinal tract, particularly the location of the pancreatic tail, which was the predilection site of splenic artery complications. The difference between CUS and CEUS may be attributed to the fact that CEUS is able to display the vessels with contrast agent injection $(33,34)$.

Early diagnosis of SAP is important in optimizing the monitoring and treatment of patients as early as possible (35-37). CECT is considered to be the standard diagnostic imaging modality for SAP (12). Diagnosis of SAP using CUS is based on indicators such as pancreatic size, PPFC, and the quality of echogenicity of the pancreatic parenchyma or fluid collection. However, CUS is unable to detect pancreatic parenchyma (38). Although the diagnosis of SAP using CUS has indirect objectives, these are not widely accepted $(39,40)$. This is because the necrosis pancreatic parenchyma can not be shown by CUS directly, and the pancreatic size, PPFC and the quality of echogenicity of the pancreatic parenchyma or fluid collection are regarded as indicators of SAP by CUS indirectly.

Diagnosis was more accurate with CEUS than with CUS. The diagnostic accuracy rates of US and CEUS for SAP in the present study were $47.7 \%$ (63/132) and 78.0\% (103/132), respectively. As demonstrated in previous studies, in terms of the assessment of pancreatic size and PPFC, the diagnostic accuracy rate for splenic vessel complications, and statistical results, CEUS was superior to US in the diagnosis of SAP $(10,14,22)$. Significant differences were observed between the results for CEUS and CUS. Since contrast agents can clearly show the microcirculation of the pancreatic parenchyma, necrosis of the pancreatic parenchyma will be clearly displayed on CEUS. Thus, pancreatic parenchymal necrosis 
should display no enhanced area following contrast agent injection (32).

In conclusion, CEUS is a reliable method for the diagnosis and monitoring of AP and SAP, and may serve as a substitute for CECT.

\section{Acknowledgements}

The authors are indebted to Professors Xia Q, Huang ZW and Tang WF (Department of Western and Chinese Integrated Medicine of West China Hospital) for providing the cases in this study. This study was supported by the nurses Lan L and Feng XY who were the operators of contrast agent injection.

\section{References}

1. Harvey C, Hart JL and Lloyd CR: Ultrasound in the acute abdomen. Br J Hosp Med (Lond) 69: M116-M119, 2008.

2. Pach R, Kulig P, Kołodziejczyk P, Szczepanik A and Sierżęga M: Ultrasonography in the diagnosis of acute abdominal disorders. Pol Przegl Chir 84: 590-600, 2012.

3. Ćwik G: Standards of the Polish Ultrasound Society - update. Pancreas examination. J Ultrason 13: 167-177, 2013.

4. Frossard JL, Steer ML and Pastor CM: Acute pancreatitis. Lancet 371: 143-152, 2008.

5. Dellinger EP, Forsmark CE, Layer P, Lévy P, Maraví-Poma E, Petrov MS, Shimosegawa T, Siriwardena AK, Uomo G Whitcomb DC, et al: Determinant-based classification of acute pancreatitis severity: An international multidisciplinary consultation. Ann Surg 256: 875-880, 2012.

6. Triester SL and Kowdley KV: Prognostic factors in acute pancreatitis. J Clin Gastroenterol 34: 167-176, 2002.

7. Bollen TL, Singh VK, Maurer R, Repas K, van Es HW, Banks PA and Mortele KJ: A comparative evaluation of radiologic and clinical scoring systems in the early prediction of severity in acute pancreatitis. Am J Gastroenterol 107: 612-619, 2012.

8. Bai Y, Liu Y, Jia L, Jiang H, Ji M, Lv N, Huang K, Zou X, Li Y, Tang C, et al: Severe acute pancreatitis in China: Etiology and mortality in 1976 patients. Pancreas 35: 232-237, 2007.

9. Gandolfi L, Torresan F, Solmi L and Puccetti A: The role of ultrasound in biliary and pancreatic diseases. Eur J Ultrasound 16: 141-159, 2003.

10. Rickes S, Uhle C, Kahl S, Kolfenbach S, Monkemuller K, Effenberger $\mathrm{O}$ and Malfertheiner P: Echo enhanced ultrasound: A new valid initial imaging approach for severe acute pancreatitis. Gut 55: 74-78, 2006.

11. Ardelean M, Şirli R, Sporea I, Bota S, Martie A, Popescu A, Dănila M, Timar B, Buzas R and Lighezan D: Contrast enhanced ultrasound in the pathology of the pancreas - a monocentric experience. Med Ultrason 16: 325-331, 2014.

12. Balthazar EJ: Acute pancreatitis: Assessment of severity with clinical and CT evaluation. Radiology 223: 603-613, 2002.

13. Faccioli N,Crippa S, Bassi C and D'Onofrio M: Contrast-enhanced ultrasonography of the pancreas. Pancreatology 9: 560-566, 2009.

14. Lu Q, Zhong Y, Wen XR, Huang ZW, Fan YT, Xia Q and Luo Y: Can contrast-enhanced ultrasound evaluate the severity of acute pancreatitis? Dig Dis Sci 56: 1578-1584, 2011.

15. Rickes S, Rauh P, Uhle C, Ensberg D, Mönkemüller K and Malfertheiner P: Contrast-enhanced sonography in pancreatic diseases. Eur J Radiol 64: 183-188, 2007.

16. Rickes S, Mönkemüller K and Malfertheiner P: Acute severe pancreatitis: Contrast-enhanced sonography. Abdom Imaging 32: 362-364, 2007.

17. Murphy KP, O'Connor OJ and Maher MM: Updated imaging nomenclature for acute pancreatitis. AJR Am J Roentgenol 203: W464-W469, 2014.

18. Windsor JA and Petrov MS: Acute pancreatitis reclassified. Gut 62: 4-5, 2013.
19. Shanaman MM, Schwarz T, Gal A and O'Brien RT: Comparison between survey radiography, B-mode ultrasonography, contrast-enhanced ultrasonography and contrast-enhanced multi-detector computed tomography findings in dogs with acute abdominal signs. Vet Radiol Ultrasound 54: 591-604, 2013.

20. Dhaka N, Samanta J, Kochhar S, Kalra N, Appasani S, Manrai M and Kochhar R: Pancreatic fluid collections: What is the ideal imaging technique? World J Gastroenterol 21: 13403-13410, 2015.

21. Bollen TL, van Santvoort HC, Besselink MG, van Es WH, Gooszen HG and van Leeuwen MS: Update on acute pancreatitis: Ultrasound, computed tomography and magnetic resonance imaging features. Semin Ultrasound CT MR 28: 371-383, 2007.

22. Golea A, Badea R, Socaciu M, Diaconu B and Iacob D: Quantitative analysis of tissue perfusion using contrast-enhanced transabdominal ultrasound (CEUS) in the evaluation of the severity of acute pancreatitis. Med Ultrason 12: 198-204, 2010.

23. Patil PV, Khalil A and Thaha MA: Splenic parenchymal complications in pancreatitis. JOP 12: 287-291, 2011.

24. Hayashi H, Beppu T, Okabe K, Masuda T, Okabe H and Baba H: Risk factors for complications after partial splenic embolization for liver cirrhosis. Br J Surg 95: 744-750, 2008.

25. Wang L, Liu GJ, Chen YX, Dong HP and Wang LX: Sinistral portal hypertension: Clinical features and surgical treatment of chronic splenic vein occlusion. Med Princ Pract 21: 20-23, 2012.

26. Heider R and Behrns KE: Pancreatic pseudocysts complicated by splenic parenchymal involvement: Results of operative and percutaneous management. Pancreas 23: 20-25, 2001.

27. Carr SC, Mahvi DM, Hoch JR, Archer CW and Turnipseed WD Visceral artery aneurysm rupture. J Vasc Surg 33: 806-811, 2001.

28. Malka D, Hammel P, Lévy P, Sauvanet A, Ruszniewski P, Belghiti J and Bernades P: Splenic complications in chronic pancreatitis: Prevalence and risk factors in a medical-surgical series of 500 patients. Br J Surg 85: 1645-1649, 1998.

29. Mortelé KJ, Mergo PJ, Taylor HM, Ernst MD and Ros PR: Splenic and perisplenic involvement in acute pancreatitis: Determination of prevalence and morphologic helical CT features. J Comput Assist Tomogr 25: 50-54, 2001.

30. Madoff DC, Denys A, Wallace MJ, Murthy R, Gupta S, Pillsbury EP, Ahrar K, Bessoud B and Hicks ME: Splenic arterial interventions: Anatomy, indications, technical considerations, and potential complications. Radiographics 25 (Suppl 1): S191-S211, 2005.

31. Koito K, Namieno T, Nagakawa T and Morita K: Splenic artery prior to rupture in the pancreatic pseudocyst: Detection by endoscopic color Doppler ultrasonography. J Ultrasound Med 15: 721-724, 1996.

32. Dietrich CF: Comments and illustrations regarding the guidelines and good clinical practice recommendations for contrast-enhanced ultrasound (CEUS)-update 2008. Ultraschall Med 29 (Suppl 4): S188-S202, 2008

33. Cai DM, Parajuly SS, Ling WW, Li YZ and Luo Y: Diagnostic value of contrast enhanced ultrasound for splenic artery complications following acute pancreatitis. World J Gastroenterol 20: 1088-1094, 2014.

34. Cai DM, Luo Y,Li YZ, Tang WF, Huang ZW and Song B: Assessing splenic vein complications in patients with acute pancreatitis using color Doppler ultrasound and contrast enhanced ultrasound. Sichuan Da Xue Xue Bao Yi Xue Ban 45: 850-853, 2014 (In Chinese).

35. Garcea G, Jackson B, Pattenden CJ, Sutton CD, Neal CP, Dennison AR and Berry DP: Early warning scores predict outcome in acute pancreatitis. J Gastrointest Surg 10: 1008-1015, 2006.

36. Ruiz R, Pozo E and Gonzáles M: Early prognostic risk factors in acute pancreatitis: Study of a representative population from Peru. Rev Gastroenterol Peru 12: 5-11, 1992 (In Spanish).

37. Horzić M, Bunoza D and Marić K: Meaning of prognostic factors in patients with acute pancreatitis. Acta Med Croatica 51: 49-52, 1997.

38. Topal NB, Kaya E, Ercan I, Pourbagher MA and Topal U: The role of Doppler sonography in predicting severity of acute pancreatitis. J Clin Ultrasound 36: 141-147, 2008.

39. Luo Y, Yuan CX, Peng YL, Wei PL, Zhang ZD, Jiang JM, Dai L and $\mathrm{Hu}$ YK: Can ultrasound predict the severity of acute pancreatitis early by observing acute fluid collection? World J Gastroenterol 7: 293-295, 2001.

40. Ripollés T, Martínez MJ, López E, Castelló I and Delgado F: Contrast-enhanced ultrasound in the staging of acute pancreatitis. Eur Radiol 20: 2518-2523, 2010. 